\title{
Implicaciones del turismo en la ecología y el desarrollo de la amazonia en Napo, Ecuador DOI: 10.22403/UQROOMX/TYP01/03
}

\author{
Justo Rojas López
}

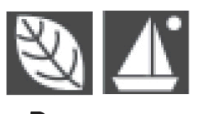

Resumen

El análisis de las implicaciones de la demanda turística en la provincia de Napo desde la perspectiva del desarrollo local revela un bajo nivel de integración entre las comunidades locales y los turistas, lo que genera insuficientes beneficios que dinamicen la economía y por tanto eleven los niveles de vida en la región. El estudio de las afectaciones ecológicas de los turistas en las zonas frágiles indica que a pesar de existir intenciones y tendencias hacia el cuidado y el respeto de la naturaleza se ejerce un fuerte desgaste del ambiente.

\footnotetext{
Palabras | Demanda turística, conservación ecológica, desarrollo local, CLAVE Napo.
}

*Universidad de Quintana Roo / jrojas@correo.uqroo.mx 
Implicaciones del turismo en

la ecología y desarrollo de la

amazonia en Napo, Ecuador

\section{Introducción}

Al despuntar la década de los setenta, la industria turística de la región amazónica acelera la transformación de su sociedad originaria. Algunas de las actividades tradicionales empezaron a ser suplantadas o suplementadas por las actividades foráneas; un nuevo ambiente se cernió sobre las comunidades locales, caracterizado en su aspecto más evidente por la constante presencia de extranjeros además de la creación y desaparición frecuente de infraestructura en función de los ciclos económicos del fenómeno.

Adicionalmente, en los últimos 10 años surgió la iniciativa del gobierno central y de diversas organizaciones no gubernamentales (ONG), sobretodo extranjeras, de convertir el turismo regional al llamado ecoturismo, que tiene como base teórico-metodológica el aprovechamiento de la naturaleza como ámbito espacial preferente, buscando siempre reducir el daño, además de incorporar directamente a las comunidades locales a la administración y disfrute de los beneficios surgidos del turismo (Morera, 1998: I I2).

Sin embargo, en la región, este nuevo fenómeno ha tenido particularidades evidentes: la incorporación de comunidades indígenas y rurales a la industria por medio de la creación de áreas rudimentarias de alojamiento y recreación, impulsadas económicamente por las ONG; la oferta de las diversas áreas protegidas como atractivos turísticos; la numerosa existencia de pequeñas empresas (tanto de dueños locales como foráneos) dedicadas al turismo de aventura, y la presencia de desarrollos turísticos con señales características de ser enclaves económicos.

Dada esta realidad palpable en la región, los habitantes locales y los estudiosos del turismo realizan cuestionamientos sociales y ecológicos a esta nueva fenomenología turística regional:

I. ¿Cuáles son las verdaderas implicaciones en la ecología regional de los sucesos provocados por el nuevo turismo, más allá de lo aparente o superficial?

2. ¿Existe la participación real de las comunidades locales en las actividades y en la repartición de beneficios del turismo? y, en general, ¿Esta participación ha implicado desarrollo social de la comunidad?

3. ¿Se cierra el círculo de la sustentabilidad de la actividad en la región?

TEORÍA Y PRAXIS

I (2005: 49-67) 
entendida como la complementación de la sustentabilidad económica (competitividad del destino), ecológica y social (Morera: 2004).

Preguntas que el presente artículo desea responder desde el análisis de la demanda turística, es decir, en el estudio de las acciones y motivaciones de los turistas visitantes de la región amazónica.

La Provincia de Napo se ubica al centro-este del Ecuador y representa la sección ecuatoriana de la amazonía estudiada, esta se conforma por los cantones de Chaco, Quijos, Archidona, Tena y Carlos Julio Arosemena Tola, con una superficie de 13.342 $\mathrm{km}^{2}$, equivalente al I I,54\% del total de la Región Amazónica Ecuatoriana (figura I). Según el último Censo de Población y Vivienda disponía para el año 200 I de una población de 79.139 habitantes, mayoritariamente rural $(67,5 \%)$, concentrada preferentemente en Tena, capital provincial, donde reside el $58,1 \%$ de la población total de la provincia.

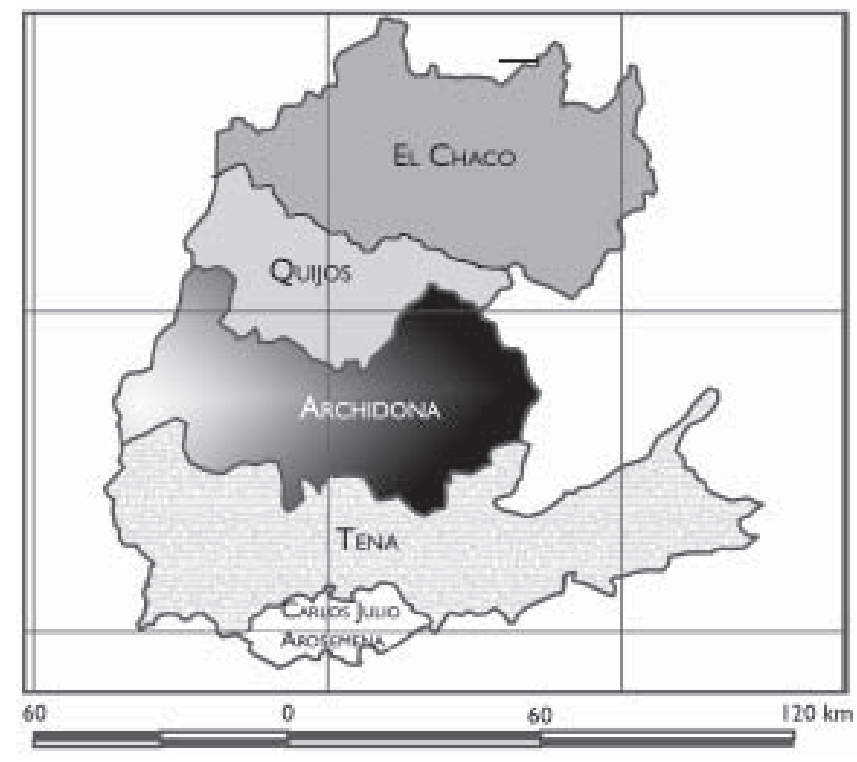

Figura I. Localización del área de estudio 
Implicaciones del turismo en

la ecología y desarrollo de la

amazonia en Napo, Ecuador

Para ello el tratamiento del tema se divide en tres partes: la primera se aboca al análisis de la relación turistas comunidad-local, en la búsqueda de la existencia de integración y complementación socioeconómica generadora de sinergias. La segunda investiga las implicaciones de las acciones y las motivaciones de los visitantes en la conservación ecológica. Y en la última se incluyen las conclusiones de los temas anteriores.

Desde el punto de vista ambiental, la provincia de Napo se localiza en la llanura amazónica, caracterizándose por la presencia de una variada vegetación. Cuenta con cuatro unidades del Sistema Nacional de Áreas Protegidas: Parque Nacional Llanganates, Reserva Ecológica Antisana, Reserva Ecológica Cayambe Coca y la Reserva de la Biosfera del Gran Sumaco.

La dinámica demográfica de la provincia ha estado fuertemente influenciada por las actividades económicas que se han establecido, la actividad petrolera desde la década de los 70; la agroexportadora de la Palma Africana, década de los 70 y 80 , el turismo y ecoturismo en la década de los 90 .

\section{Metodología}

\section{a) Estudio de gabinete}

Se efectuó una revisión bibliográfica de la teoría general del turismo, afectaciones del turismo, turismo sustentable y ecoturismo, para lo cual se tomaron las sugerencias de Wearing y Neil (1999), Mathieson y Wall (1990), Rojas (2000) y Morera (1998). Además se analizaron y aceptaron para el estudio las participaciones de la comunidad local en el desarrollo; las ideas de Morera (2004), Pérez (1999), Davison (1993) y Mathieson y Wall (1990). En el diagnóstico de la situación del turismo en la región amazónica, se toman como guías las investigaciones realizadas por el Ministerio de Turismo de Ecuador (2003), ECORAE (2002 y 2003). Por último, para la selección metodológica del muestreo y la elaboración de instrumentos de levantamiento de información se siguen las consideraciones de Fischer (1995) y Morera (2004).

\section{b) Estudio de Campo}

Se realizó un estudio de caracterización de los visitantes, cuya primera etapa consistió en obtener una muestra estadística de I 40 turistas de un universo (Fischer, 1995: 56-63) determinado en función del número de visitantes a la 
zona en los últimos 12 meses (I 5000 visitantes), según el Ministerio de Turismo de Ecuador. El segundo paso consistió en una visita de reconocimiento del sitio de estudio para definir estrategias y herramientas de levantamiento. Después se establecieron las localidades donde se realizaron las encuestas:Tena Misahualli Pano, Puerto Napo,Ahuano, por ser los mercados consolidados del turismo provincial. Enseguida se realizó un levantamiento de encuestas que abarcó a 120 visitantes de la provincia, debido a cuestiones de costo y accesibilidad a sus sitios de reunión, sin embargo, esta falta afecta poco a la representatividad. Una salvedad más fue que las encuestas fueron posteriores a la visita del turista a la región, con el propósito de recabar información pertinente a su estancia en el sitio. Además, se aplicaron en diferentes puntos clave caracterizados por ser lugares de alta afluencia de visitantes, tales como estaciones de autobuses, aeropuertos, parques, sitios de hospedaje y lugares de tránsito frecuente.

Los resultados fueron depurados a fin de minimizar sesgos, y se tabularon para permitir la realización de cruces de variables que facilitaron la identificación de hallazgos y tendencias.

\section{Resultados}

\section{a) Relaciones turistas y comunidad local}

En particular, la sociedad de Napo ha venido experimentando la presencia de turistas desde hace aproximadamente quince años, con un incremento en la década de 1990, lo que puede significar, como señalan Morera (2004: 24) y Wearing y Neil (1999: I48- I49), tanto problemas como beneficios para la comunidad.

Es necesario entonces dilucidar hacia cuál de estas dos tendencias se carga la situación relacional turistas-comunidad local. Para ello se identificaron ciertas formas de interacción que, basadas en las ideas de De Kadt (1979: 50, citado por Mathieson y Wall, 1990: 173) indican diferentes niveles de interacción y entendimiento entre los dos actores, las cuales fueron: el consumo en tiendas locales (baja sinergia), la conversación callejera (baja sinergia), los almuerzos con pobladores (alta sinergia), los viajes compartidos en transporte público (baja sinergia) y, finalmente, la creación de lazos de amistad (alta sinergia). 
Implicaciones del turismo en

la ecología y desarrollo de la

amazonia en Napo, Ecuador

Los resultados muestran niveles de incidencia similares en las interacciones de la demanda turistica con la comunidad de Napo, tal como lo observa en la gráfica I.

Gráfica I.Tipos de interaccion turistica comunidad local

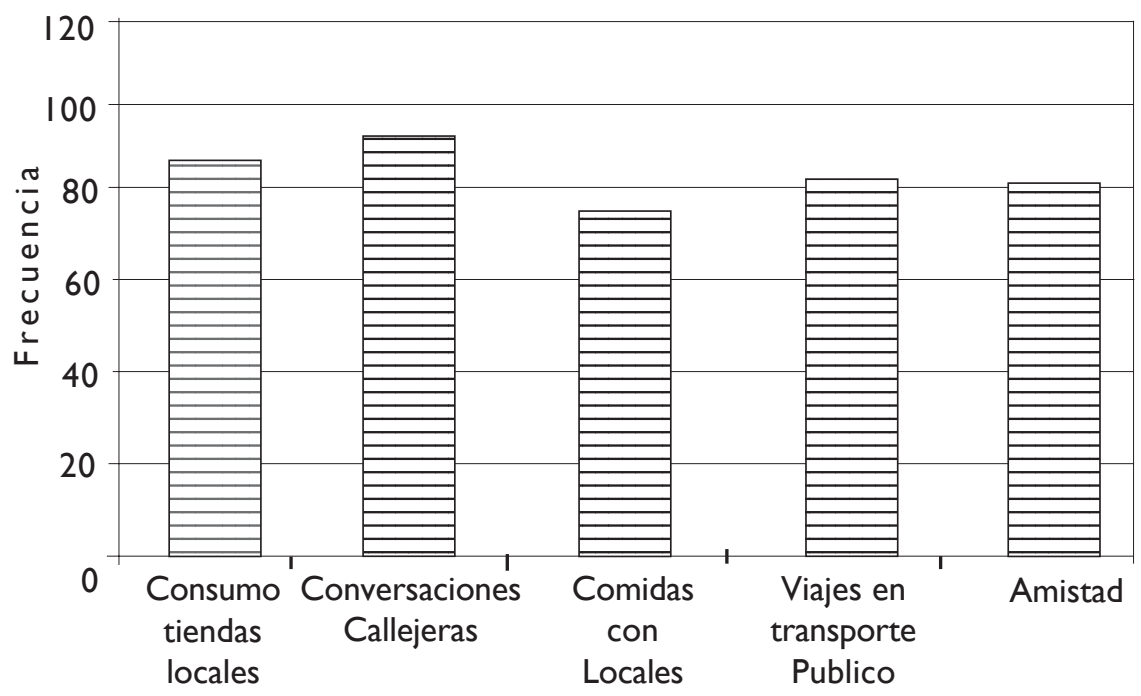

Tipo de Interacción

Sin embargo, a detalle se nota que es la conversación callejera con los habitantes la forma de contacto más practicada por los turistas (con 92 frecuencias), seguida por el consumo en tiendas locales y los viajes en transportes (89 y 83 frecuencias, respectivamente) que, como se mencionó, implican en conjunto bajos niveles de integración entre los turistas y la comunidad local. Se encuentra, además, que los factores, como amistad y almuerzo con pobladores locales, que contemplan conjuntamente una mayor integración entre los turistas y las comunidades locales, tienen niveles inferiores de repetición ( 81 y 75 , respectivamente) al grupo anterior, entre los turistas encuestados.

Esta inclinación hacia las formas de relación de poca integración entre los turistas y la comunidad local ha influido en la reconfiguración espacial 
de las poblaciones y de sus actividades económicas, que en el caso de las comunidades indígenas es más notorio, debido a que ha contribuido a la comercialización de sus costumbres y transformación o "modernización" de sus espacios habitacionales, en tanto que en las ciudades, los cambios han sido más en el aumento de las actividades microempresariales. A la vez, se nota que este encuentro de la comunidad local con el distinto ha propiciado, debido a la necesidad económica, la creación de una conciencia de respeto al ambiente, pero que por fundarse en la conveniencia parece ser poco estable.

\section{b) Interacciones económicas}

En esta sección se intenta esclarecer cuáles son los beneficios emanados del turismo en Napo, tomando en cuenta que desde la teoría se plantea, en su aspecto más amplio, mejorar la calidad de vida de las comunidades locales (Morera, 1998: 5); y más detalladamente, la generación de empleos (Pérez, 1999: 123; Davison, 1993: I58) y el incremento de la demanda por hospedaje y alimentos, que aumenten la generación de nuevas empresas locales (Wearing y Neil, 1999: 396). Por tanto, estos son los aspectos que deben ponderarse en la evaluación socioeconómica del turismo de Napo.

El primer elemento de medición utilizado es la forma como la demanda turística organiza sus viajes, la cual sirve para identificar las vías y los beneficiarios de las remesas económicas que los turistas deben distribuir en sus intercambios comerciales, además de indicar el grado de participación de los habitantes locales en esos intercambios, por último, describe si el impacto del turismo se inicia fuera o dentro de la región y si éste se materializa en los gastos que el turista realiza en la comunidad local (Rojas, 2000: 49).

Este indicador arroja que la composición de los viajes de la demanda turística en Napo es dominada por una sola forma de organización: el viaje por cuenta propia sin ningún tipo de paquete, tal como lo muestra la gráfica 2 .

Este primer tipo de organización de viaje concentra $66 \%$ de la demanda turística, seguido por el paquete completo organizado en el país de origen, por el paquete completo comprado en Ecuador, y por último, el paquete abierto, comprado en el lugar de origen del visitante. Resultados que coinciden fuertemente con los registrados por ECORAE, que identificó 70\% de los visitantes en las formas de viaje sin paquete (ECORAE, 2003). 
Implicaciones del turismo en

la ecología y desarrollo de la

amazonia en Napo, Ecuador

Gráfica 2. Formas de organizacion del viaje de los turistas.

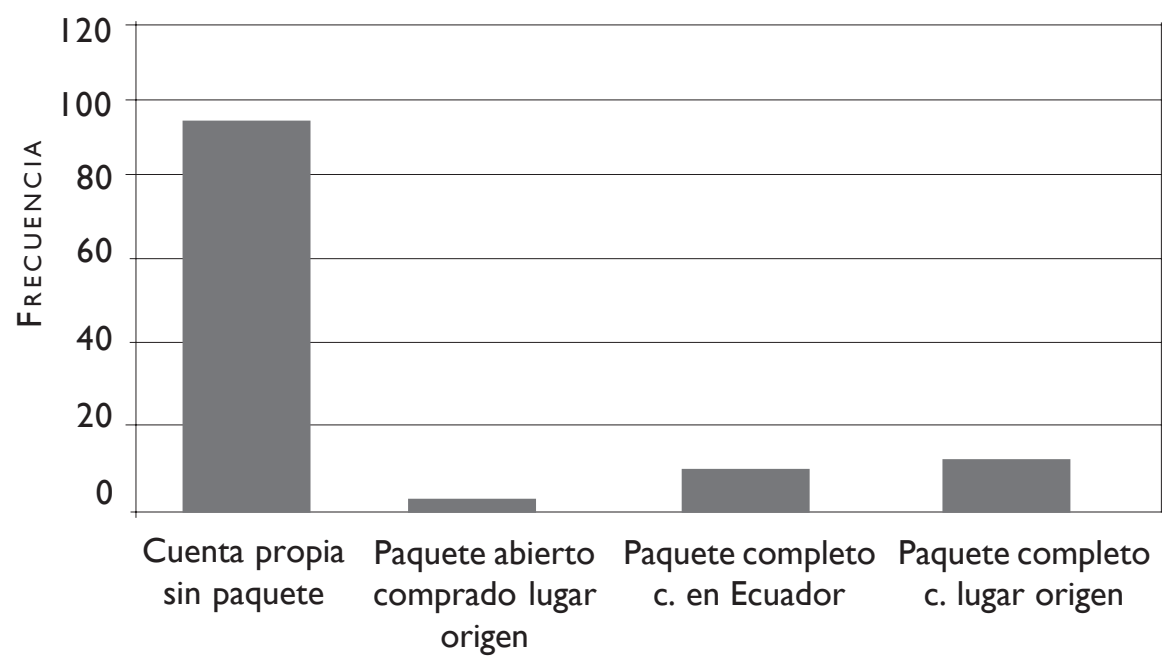

La tendencia hacia los viajes por cuenta propia potencia la posibilidad de una mayor socialización de los beneficios dentro de las comunidades receptoras, dada la mayor libertad de establecer contactos con las comunidades, en comparación con los turistas dependientes de algún tipo de paquete que se ven circunscritos a itinerarios forzosos; sin embargo, estos últimos se caracterizan por realizar un mayor gasto en su visita, además de tener una frecuencia de retorno más alta que los turistas libres (ibid.).

Otro de los elementos que ayuda a medir el impacto económico del turismo en las comunidades receptoras es el consumo de los turistas en los comercios locales, debido a que es un parámetro de medición de la socialización de los beneficios económicos emanados de ella. Se evalúan desde dos perspectivas: la cantidad y calidad de utilización de los comercios, con el fin de develar las posibles contradicciones entre los índices de medición y la realidad imperante en la región.

De esta manera se captó que $45 \%$ de los visitantes en Napo realizan compras en las tiendas de las comunidades locales, lo que señala que menos de la mitad de los turistas retribuyen algún beneficio económico a estos comercios. Lo que al añadirse a la intensidad de compra (gráfica 3) indica que 
Gráfica 3. Intensidad de consumo de turistas en tiendas locales.

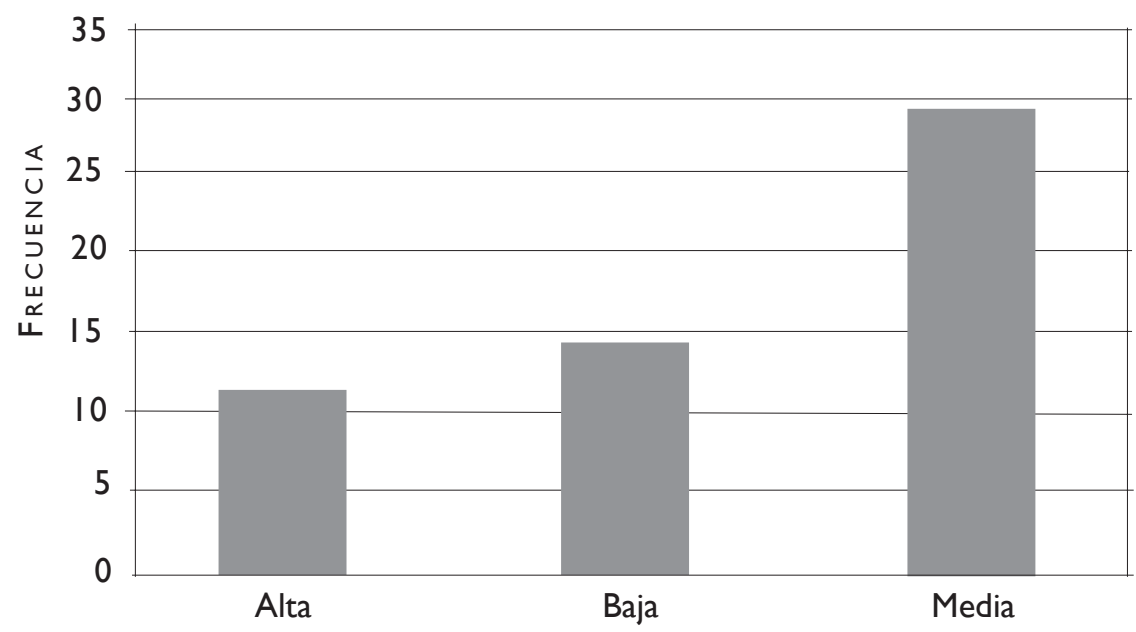

de este $45 \%$ la mayoría realiza compras en frecuencia media (53.7\%), seguida por la baja intensidad de compras (29.3\%), y por último, la alta frecuencia con el $20.3 \%$ del total de turistas, lo que demuestra en la realidad que el intercambio comercial realizado por turistas representan menos de un sexto del total de visitantes a la región. Al considerar además los salarios que los turistas devengan (que da una idea de su capacidad de compra) se encuentran que mayoritariamente los ingresos de estos compradores se ubican en el estrato salarial de 250 a 500 dólares, seguido por los que están entre I 00 I y 2500 dólares, siendo el tercer escaño los que tienen ingresos menores a 250 dólares. Lo anterior implica que los turistas compradores en tiendas locales se encuentran entre los ingresos medios a bajos, siendo menos frecuentes los que se hallan en los niveles más altos (2 50I-5 000, 500 I o más) (gráfica 4).

El resultado del enlace entre las anteriores variables muestra que si bien existe una mayoría absoluta de turistas viajando por cuenta propia a la provincia, menos de la mitad gasta en establecimientos locales; además, que estos últimos se distinguen por realizar compras con una incidencia media a baja y porque sus gastos se rigen por presupuestos con las menores capacidad adquisitivas. Esta situación ha provocado que en Napo la mayoría de los negocios locales relacionados con el turismo sean microempresas poco 
Implicaciones del turismo en

la ecología y desarrollo de la

amazonia en Napo, Ecuador

eficientes, sin la real posibilidad de crecimiento y desarrollo, lo que implica, dado el carácter de principal actividad económica que tiene el turismo para la comunidad, una economía local con una baja dinamicidad, es decir, una alta tasa de desempleo y de cierre de negocios, bajo poder adquisitivo y un PIB por debajo de la media nacional (ECORAE, 2003).

Gráfica 4. Salario en dólares de turistas consumidores en tiendas locales

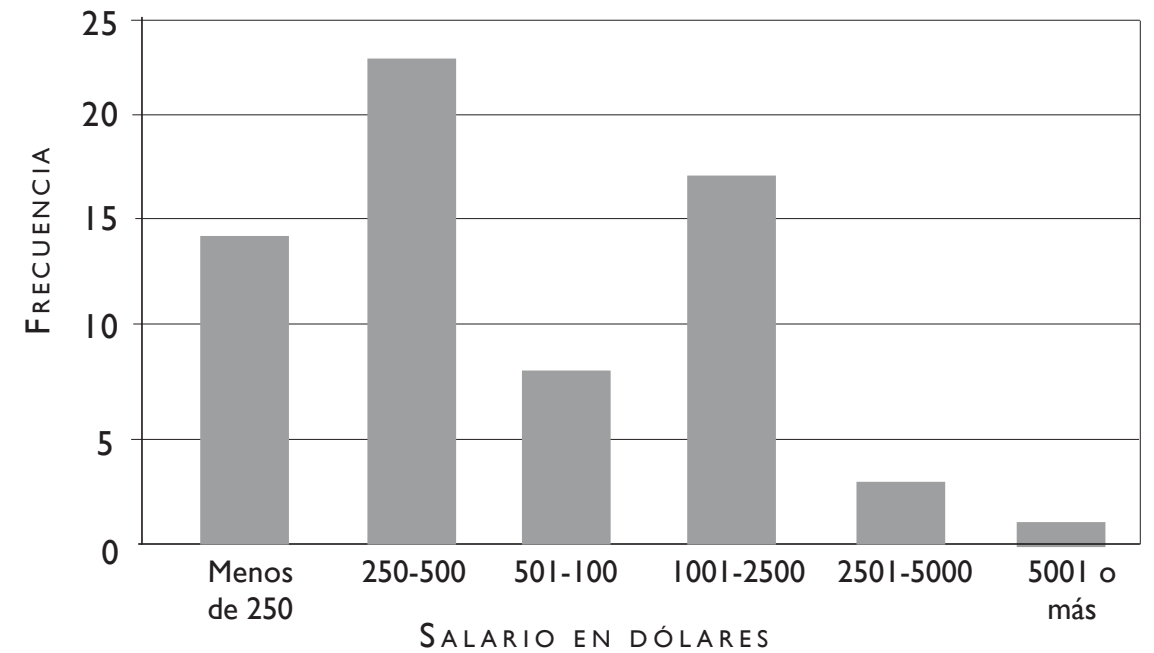

\section{c) Implicaciones en la conservación ecológica}

Los impactos ambientales están ligados necesariamente a las acciones de los diferentes sujetos del ámbito turístico. Por tanto, es imperativo primero conocer el perfil y las características tanto de los receptores como de los visitantes de una región, para determinar sus niveles de intervención en el daño ecológico y ponderar su grado de responsabilidad (Morera, 1998: II0, 35). Tarea que para esta investigación, encargada de la demanda turística, se basa en el análisis de las motivaciones y las actividades de los visitantes como una de las causas principales de la degradación de los recursos y la pérdida de la biodiversidad.

Para identificar cuáles de esas actividades y motivaciones permiten establecer los niveles en que los visitantes realizan turismo responsable 
ambientalmente y cuáles implican destrucción en los ambientes naturales, se consideraron las ideas de Wearing y Neil como guías normativas, estas sugieren los elementos de observación, éticos y experimentales, que corresponden a las motivaciones naturales de la visita, las actividades conservacionistas y de rehabilitación de las zonas degradas, y la creación de conciencia ecológica, como los agentes primordiales del turismo ecológico, ya que estos comprenden un menor grado de impacto y una contribución a la conservación del entorno natural (Wearing y Neil 1999: 27); y se excluyeron al resto de las motivaciones de estas facultades conservacionistas por considerarlas perjudiciales para el ambiente. Por tal motivo, se analiza primero la variable motivaciones de la visita como uno de los elementos para la búsqueda del turismo responsable ecológicamente en la provincia de Napo, específicamente como una señal de la conciencia previa del turista y por tanto, una orientación rectora de sus presentes y futuras acciones.

Gráfica 5. Motivaciones de viaje de los turistas.

\section{$0.65 \%$ Familiares}

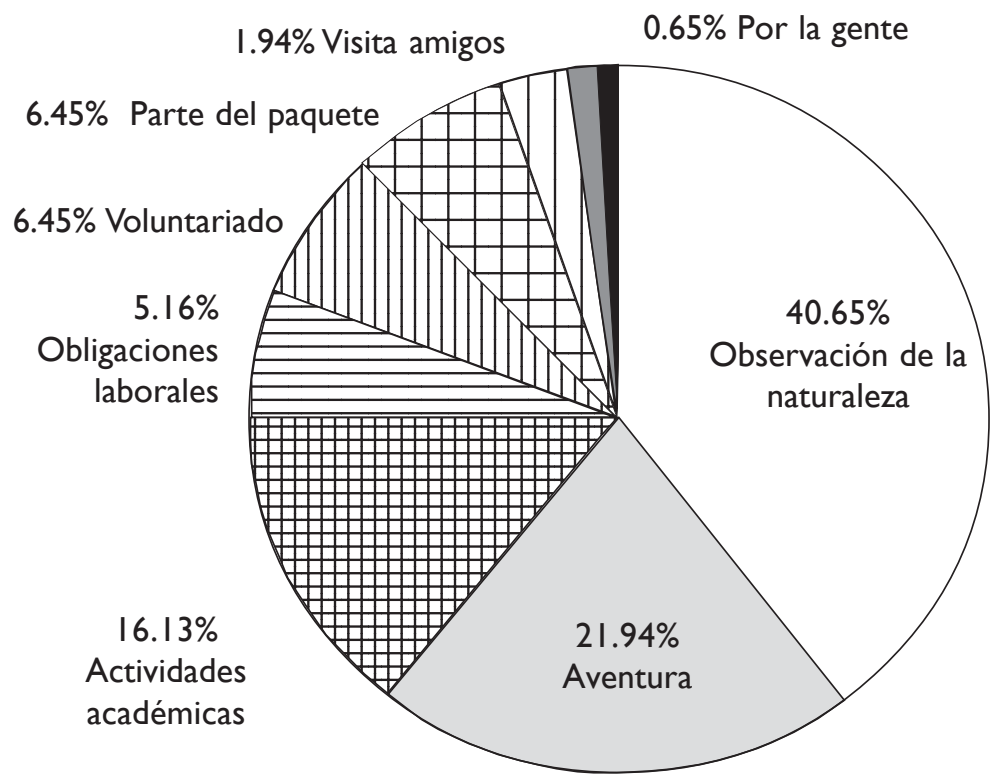


Implicaciones del turismo en

la ecología y desarrollo de la

amazonia en Napo, Ecuador

Los resultados (gráfica 5) muestran que las principales motivaciones de los turistas para realizar su visita son: la observación de la naturaleza, $40.65 \%$ de los visitantes; seguida por la aventura, con $21.94 \%$, y en tercer puesto las vistas a grupos indígenas, con $16.13 \%$. En estas tres primeras motivaciones se distingue que dos de ellas, la primera y la tercera, implican orientaciones definitivas hacia actividades de conservación ecológica, ya que ponderan la conservación natural y la educación sobre la utilización superficial del ambiente. Por el contrario, la segunda actividad se distingue como disfrute superficial de la naturaleza, debido a que la involucra sólo como un requisito para su verdadero propósito, la experiencia fuera de ámbitos extracotidianos y no la naturaleza en sí, por lo que puede significar en algún momento acciones destructivas. Otros aspectos a destacar son: que se encuentra una considerable motivación al voluntariado en ayuda a la naturaleza y las comunidades locales (con 6.45\%) que se inscriben dentro de las actividades éticas que los turistas realizan y que son parte de los preceptos del ecoturismo (Wearing y Neil, 1999: 14).

Se observa entonces que $63.23 \%$ de las motivaciones de viaje tienen la impronta del ecoturismo, lo que sugiere que los turistas tenían previo a su llegada ideas de conservación, es decir, una mentalidad de cuidado ambiental.

Un aspecto que mejora esta determinación, parcial hasta ahora, de las posibles implicaciones ecológicas de la presencia de turistas, son las actividades que realizan en la provincia; estas van más allá de analizar las intenciones de viaje de los turistas, abocándose a las acciones y sus posibles impactos en la naturaleza. Para ello se sigue la guía de las directrices teóricas anteriores, que establecen la conservación alrededor de actividades que procuren por el aprendizaje ecológico, y prioricen la naturaleza como parte primordial de la actividad-experiencia de los turistas.

El análisis de las actividades de los turistas y su relación con la conservación ecológica muestran (gráfica 6) que las acciones de la demanda turística relacionadas con el turismo ecológico son: observación de flora y fauna con un 13.69\%; seguido por caminatas, $12.55 \%$, y las visitas a áreas protegidas, 12.34\%; adicionalmente, dentro de las actividades que tienden a la conservación ecológica se encuentran las visitas a comunidades indígenas, que representan $10.58 \%$. Lo cual indica que en total $49.15 \%$ de los turistas realizan actividades turísticas que se consideran conservacionistas. Esto plantea 
Gráfica 6. Actividades turisticas de los visitantes de la porvincia

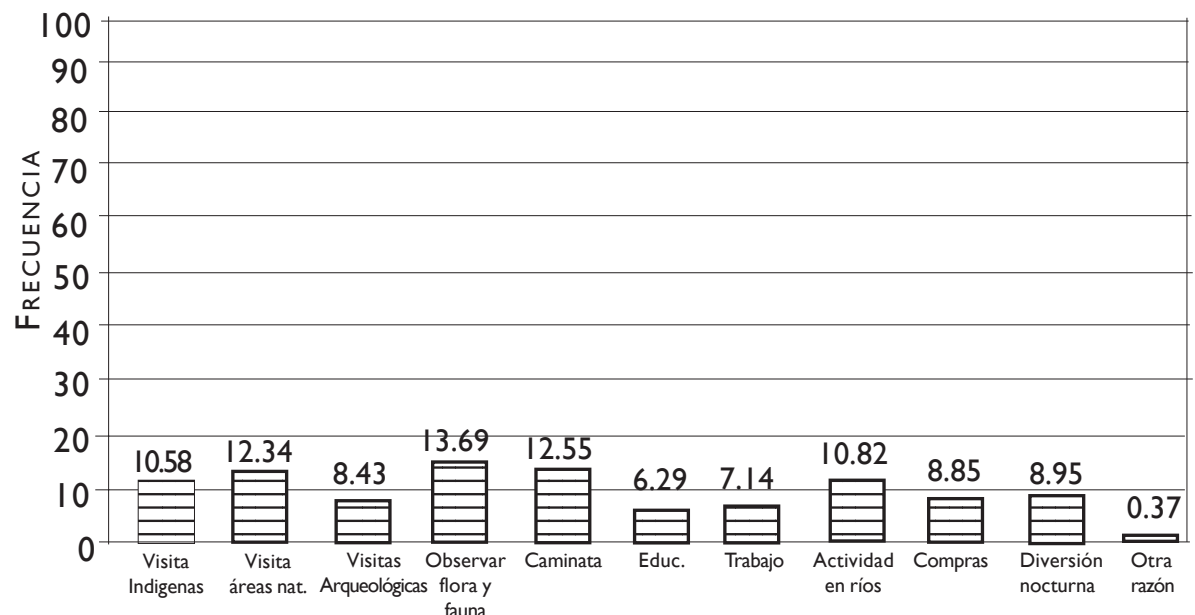

que menos de la mitad de los turistas realizan este tipo de actividades, lo que tiene serias afectaciones al entorno y muestra una predominancia del tradicional modelo de objetivización de la naturaleza.

Pero por otro lado indica también la existencia de una relevante y emergente gama de acciones ecoturísticas que tienen implicaciones en el actual sistema turístico, ya que al ser un importante mercado de actividades que implican conservación, necesariamente modifica las estructuras de la oferta turística con las que tuvo contacto, para ajustarlas a las exigencias del cuidado ambiental que implica la nueva perspectiva.

Una vez establecido que existen en la zona una cantidad considerable de turistas con actividades conservacionistas es necesario explorar los elementos que los caracterizan a detalle (nivel educativo, incidencia de visita, número de personas por grupo visitante y la creación de conciencia ecológica) debido a que estos son parte esencial en una medición más compleja y acertada de los grados de impacto ecológico sobre la región y sobre todo en las áreas consideradas frágiles (áreas protegidas y comunidades indígenas) donde se concentra esta parte del análisis.

La incidencia de visitación, en conjunto con la educación, es una de las formas escogidas para determinar condiciones económicas y de conciencia 
Implicaciones del turismo en

la ecología y desarrollo de la

amazonia en Napo, Ecuador

Gráfica 7. Frecuencia, intensidad de visita y nivel educativo de turistas visitantes a las comunidades indígenas.

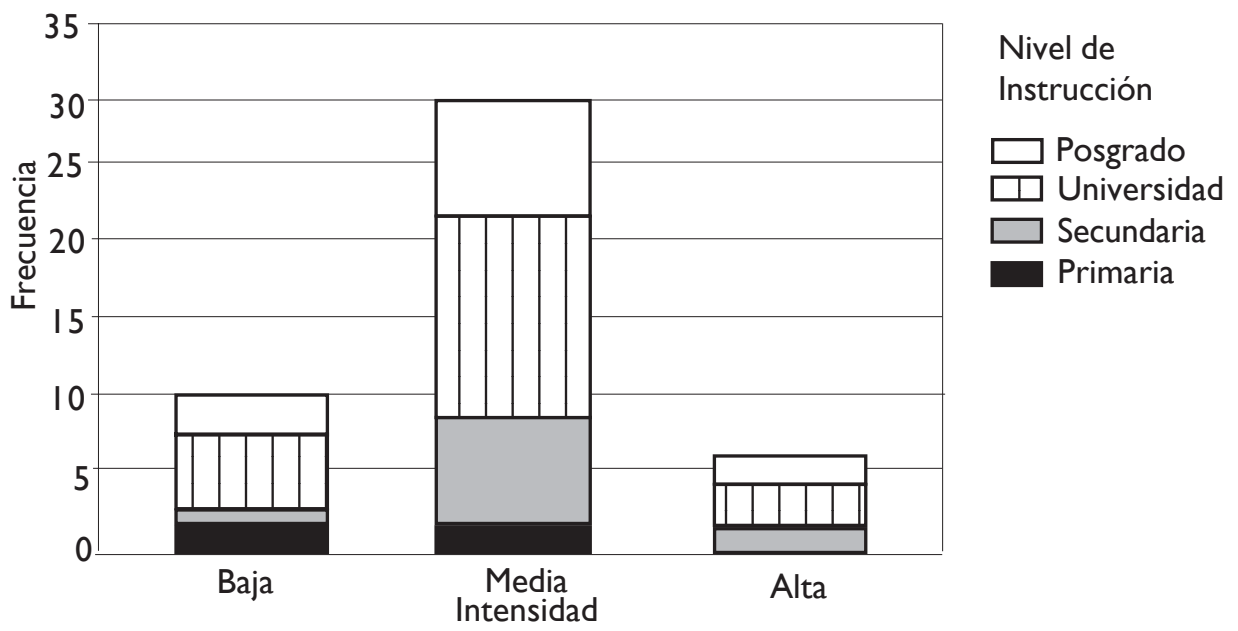

de conservación, como el binomio que permite establecer de manera cualitativa los posibles efectos de las actividades de la demanda turística en la provincia (Morera, 1998: 48, 64). La figura 7 muestra que el nivel medio de intensidad de visitación a las comunidades indígenas es el más alto de los realizados por los visitantes, conformado por un componente mayoritario de turistas con grado de instrucción universitaria, seguido por los de posgrado y por los turistas con nivel de secundaria, como los principales. El segundo grado de incidencia de visitación encontrado es el nivel bajo, que se conforma primero por turistas con grado universitario, seguido por los posgraduados y en mínima cantidad el resto de los niveles educativos. Como último resultado está el alto nivel de repetición de vistas a las comunidades indígenas, que aglomera preponderantemente a turistas de nivel universitario, seguido por los turistas a nivel de posgrado, y al final, los instruidos a nivel secundario.

Destaca de lo anterior, primero, la media-baja recurrencia de visitantes, además que en todas las frecuencias de visita a comunidades indígenas, los niveles educativos universitarios y de posgrado de los turistas son los detentadores de los más altos niveles de participación, lo que tiene influencia 
en los grados de conservación, debido a que en conjunto sugieren un menor y mejor contacto e interacción. Sin embargo, es necesario complementar con otras variables tanto de cantidad como de calidad de la visita.

Como parte de la respuesta a esta exigencia, se utilizó la cantidad de visitantes por grupo como un elemento complementario para medir un aspecto faltante: la presión de carga sobre las áreas frágiles (áreas protegidas y comunidades indígenas) en cada visita, información necesaria dado que los grupos de turistas, aun con conciencia ecológica, pueden provocar daños ambientales, según los conservacionistas, parecidos a los causados por los turistas convencionales (ibid: 235). El análisis de esta variable indica (gráfica 8) que las comunidades indígenas reciben grupos de turistas con una aglomeración global entre I y 29 integrantes. Destacan los grupos de visitantes formados por 2 integrantes ( 18 observaciones), seguidos por los de I visitante (II) y los de 3,4 y 5 integrantes (8, 7 y 6 personas respectivamente), del resto se nota que son las agrupaciones de 20 y 21 integrantes las que tienen cantidades superiores a 2 personas por grupo.

Gráfica 8. Cantidad de grupos de turistas y su intensidad de visita a comunidades indígenas Napo.

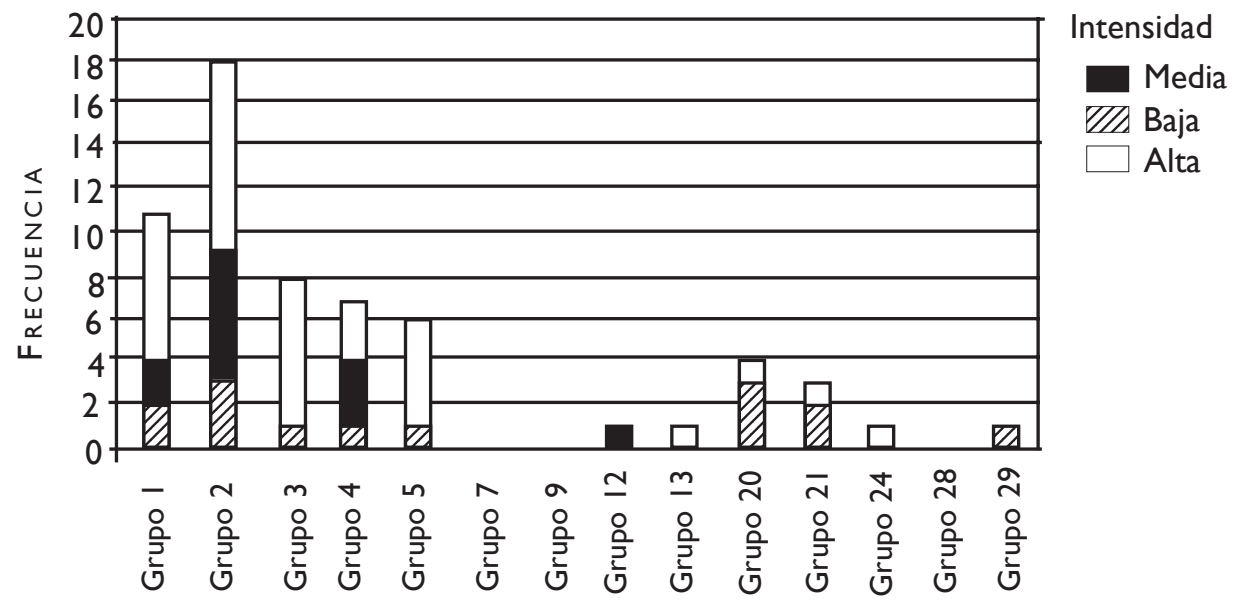

Numero de turistas por grupo 
Implicaciones del turismo en

la ecología y desarrollo de la

amazonia en Napo, Ecuador

Lo anterior muestra que las visitas de los turistas a las comunidades indígenas se realizan preponderantemente (81.9\%) en grupos de 5 y menos visitantes. Sin embargo, es relevante que en los grupos mayores (20, 2I, 24 y 29 turistas) lo realicen entre I, 2 y 4 unidades, debido a que puede representar una cantidad importante de turistas por visita (I20, 63, 24 y 29 respectivamente). Acarreando con ello posibles daños en el equilibrio de las comunidades, que pueden incrementarse considerando que en la zona no existe un control de visitación, por lo que suele suceder que las comunidades no sean visitadas por un solo grupo mesurado y alternado, sino que éstos se lleguen a superponer con otros que pueden tener elevadas concentraciones, lo que implica un fuerte desgaste.

Adicionalmente, los grados de intensidad señalan que los grupos realizadores de esta actividad la efectúan con mediana regularidad lo que ha incrementado los daños a las comunidades receptoras, que se distinguen por: un cambio en sus costumbres de organización debido al surgimiento de comités impuestos por órganos externos que suplantan a los tradicionales;

Gráfica 9. Cantidad de grupos de turistas y su intensidad de visitación a áreas naturales protegidas.

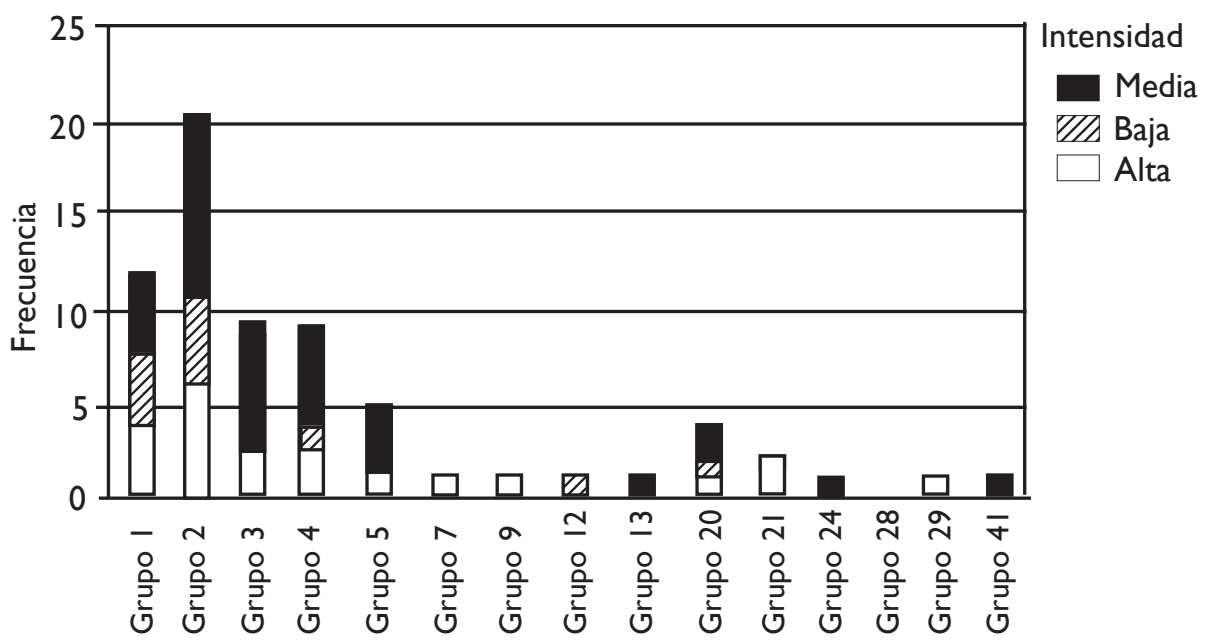

Numero de turistas por grupo

TEORÍA Y PRAXIS I (2005: 49-67) 
modificaciones en su relación con la naturaleza, dado que ha intervenido paulatinamente una lógica de utilización económica de la naturaleza que ha desplazado a su milenaria divinización y un enturbamiento de las relaciones sociales, como son las constantes disputas entre las distintas comunidades, causadas por la fuerte competencia y decadencia del mercado turístico a microescala. Por su parte, el análisis ecológico del turismo en las áreas naturales protegidas opta por buscar la misma mecánica causal de daños del caso anterior. En esta variable se encontró (gráfica 9) que en la provincia de Napo, los turistas mayoritariamente vistan las áreas protegidas en grupos de dos personas (2I), seguido por los visitantes individuales (I2), los grupos de 3 y 4 (8) y los de 5 turistas. Del resto se nota que sus concentraciones se mueven entre $I$ a 4 turistas, siendo las agrupaciones de 20 y 21 las que alcanzan entre 2 y 4 repeticiones. Además que los niveles educativos de sus integrantes se rige, al igual que la anterior medición, por los grados universitarios y de posgrado $(73 \%)$.

Esto indica, en concreto, que los grupos preponderantes se sitúan entre I y 5 integrantes considerados de baja concentración, además, que los grupos medios entre 7 a 13 integrantes, suelen tener una repetición casi nula en su visita a las áreas protegidas, y por último que los grupos entre 20 y $4 \mathrm{I}$, considerados de alta aglutinación tienen entre I y 4 frecuencias. De esto se infiere que si bien son los grupos de menor concentración los dominantes, los grupos de alta aglomeración pueden ejercer una influencia determinante, ya que sus valores, al ser multiplicados por sus repeticiones, muestran sus verdaderos impactos. Así se observa que las agrupaciones de 20 y 21 integrantes suelen introducir a las áreas protegidas entre 120 y 63 visitantes por cada asistencia, lo que puede significar daños considerables en el entorno aun cuando en la normatividad ecuatoriana existe limitación de carga en la visitación, que se rige de acuerdo con la categoría del área y sus zonas internas.

Ahora bien, al relacionar estas aglomeraciones con la medición de la intensidad de visitación para áreas protegidas se nota que los posibles daños se pueden incrementar especialmente en los grupos de alta concentración (20, 2I, 24, 29 y $4 \mathrm{I}$ turistas) debido a que además de visitar en altos conglomerados las áreas protegidas, estas visitas se realizan mayoritariamente de manera regular (grupos 20, 21,24 y 41 ) e incluso de manera frecuente (grupo 29) lo que eleva exponencialmente el desgaste de las reservas. 
Implicaciones del turismo en

la ecología y desarrollo de la

amazonia en Napo, Ecuador

\section{Conclusiones}

Existen varios elementos que distinguen los procesos turísticos que involucran el desarrollo social y la conservación ecológica acaecidos en la provincia amazónica de Napo, los cuales se presentan en los siguientes puntos clave:

- El turismo de la provincia amazónica del Napo se distingue por una relación de baja complementación entre los turistas y la comunidad local, aun cuando los visitantes están poco controlados en sus viajes por compañías extranjeras. Por su parte, las comunidades receptoras consideran a los turistas como un objeto necesario para la obtención de beneficios.

- Los beneficios económicos para las comunidades locales son bajos en cantidad y calidad, por tanto, insuficientes para un reparto en la totalidad de la economía local y menos aún para multiplicar sus efectos en el resto de la economía regional.

- Existe una creencia general del turista visitante a la región amazónica del Napo de que su presencia y sus actividades proveen un beneficio a la comunidad local

- La demanda turística tienen como propósito inicial el disfrute de la naturaleza bajo dos directrices: la primera, mayoritaria, que considera la experiencia y aprendizaje de ella y la segunda que considera el ambiente como objeto de disfrute. Lo que indica una conciencia inicial hacia el respeto de la naturaleza.

- Si embargo, no existe una total correspondencia de las motivaciones con la realidad, ya que las acciones de los turistas realizan en la región corresponden en menos de la mitad a actividades relacionadas con la conservación ecológica.

- En las zonas frágiles (naturales e indígenas) existe en general una situación contradictoria entre la pretensión de respeto y cuidado ecológico y su realización de facto, debido a que las prácticas de los turistas cargan sobre las áreas una fuerte tensión y variados conflictos.

- En la región amazónica del Napo se observa un incipiente turismo con visión ecológica, sin embargo, ésta no se consolida porque no existe una implicación total de la comunidad local en la administración y 
recepción de los beneficios, además de que el ambiente carece de la falta de condiciones para su conservación.

\section{Fuentes consultadas}

Davison, R. (1993) Tourism. Londres: Pitman Publishing, p. I 58.

ECORAE (2002) Zonificación ecológica-económica de la amazonía ecuatoriana.

Disco compacto. Quito: Grafic Art.

------ (2003) Zonificación ecológica-económica de la amazonía ecuatoriana. Disco compacto. Quito: Grafic Art.

Fischer, L. (1995) Introducción a la investigación de mercados. México: Mc Graw Hill, p. 56-63.

Mathieson, A y G. Wall (1990) Turismo: Repercusiones económicas, físicas y sociales. México:Trillas, p. 173.

Ministerio de Turismo de Ecuador (2003). Sistema de estadísticas turísticas del Ecuador. Disco compacto. Quito, ISSN-I390-2229.

Morera, C. (1998) Turismo sustentable en Costa Rica. Quito, Ecuador:AbyaYala, p. 5-235.

-------- (2004) "Ecoturismo y desarrollo local en la península de Osa, Costa Rica”. Revista Geográfica de América Central, (en prensa) p. 24.

Pérez, M. (1999) La guía del ecoturismo. Barcelona, España: MundiPrensa, p. 123.

Rojas, L. (2000) “Ecoturismo en Costa Rica”. Ecoturismo. Revista. Núm. 35, p. 49. Wearing, S y J. Neil (1999) Ecoturismo: Impacto, tendencias y posibilidades. Madrid: Síntesis, p. I4-396. 


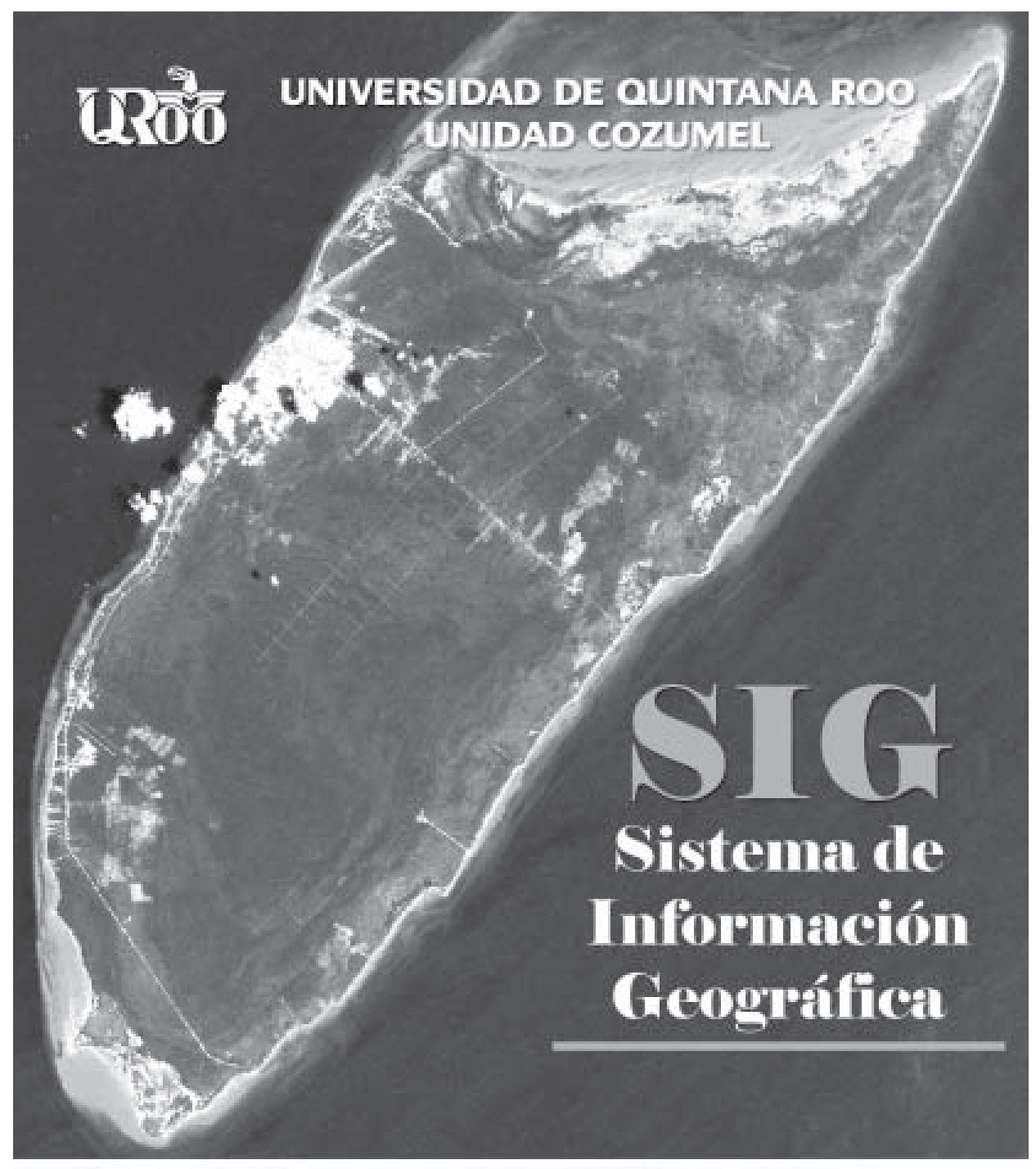

El Sistema de Información Geográfíca de la Universidad de Quintana Roo, con apenas tres af́os de servicios, ha tenido importantes logros en la adquisición y creación de software y hardware.

Entre las ventajas que podria tener este Sistema de Información Geográfica para una comuna como la de Cozumel figura el manejo del Catastro, de la información sobre negocios, de Proteceión civil, de Desarrollo Utbano, de Protección al Medio ambiente, entre otros grandes rubros. 